\title{
Bupropion SR als Unterstützung in der Raucher- entwöhnung rückfälliger Raucher: Ergebnisse einer offenen multizentrischen Studie in Deutschland
}

\author{
L. Bergmann \\ W. Warncke \\ M. Herschel
}

\author{
Bupropion SR for Weaning from Smoking in Relapsed Smokers: \\ Results of an Open Multicentre Trial in Germany
}

\section{Zusammenfassung}

Hintergrund: Durch den Suchtcharakter des Rauchens kommt es bei der Behandlung der Nikotinabhängigkeit oft zu Rückfällen. Patient und Arzt unternehmen häufig in therapeutischem Nihilismus keine weiteren Entwöhnungsversuche. Nach ermutigenden Ergebnissen zweier kontrollierter Studien in den USA und Kanada bei Patienten, die nach einer Raucherentwöhnung mit Bupropion SR $\left(\right.$ Zyban $\left.^{\circledR}\right)$ rückfällig und erneut mit Bupropion SR behandelt wurden, legen wir hier die Resultate einer Studie in Deutschland unter den Bedingungen und Zeitlimitierungen des Praxisalltags vor. Methode und Patienten: An dieser offenen Studie nahmen 365 Raucher (mindestens 20 Zigaretten/d) ohne relevante Vorerkrankungen in 62 Studienzentren teil, die unter Bupropion SR oder einem Nikotinersatz-Therapeutikum rückfällig geworden waren. Bupropion SR $(300 \mathrm{mg} / \mathrm{d})$ wurde nach dreitägigem Einschleichen mit der halben Dosis über 7 Wochen gegeben. Die Patienten wurden über insgesamt 6 Monate in 4 Visiten mit motivierenden Gesprächen nachverfolgt. Ergebnisse: 55,4\% der Patienten waren 1t. Fagerström Tolerance Questionnaire stark oder sehr stark nikotinabhängig (Score 6-11). Die kontinuierliche Abstinenzrate von Woche $4-7$ betrug 29,6\% (ITT), die Punktprävalenzrate in der Woche 26 30,5\% (ITT). Bei den erneut rückfälligen Rauchern war noch nach 26 Wochen der tägliche Zigarettenkonsum deutlich geringer (durchschnittlich 10,3 versus 26,9 Zigaretten/d vor der Behandlung). Das Nikotin-Craving sank bis Woche 6 auf ein niedriges Plateau ab. Bupropion SR wurde gut vertragen, 9,7\% der Patienten (ITT) brachen die Studie wg. unerwünschter Ereignisse ab. Die häufigsten Nebenwirkungen waren Schmerzen des Bewegungsapparates und

\section{Abstract}

Background: After initially successful smoking cessation the majority of patients relapse which stresses the addiction character of nicotine dependence. The patient and the physician, in therapeutic nihilism will not make another attempt for smoking cessation. In two randomised, double-blind, placebo-controlled trials in the US and Canada relapsed smokers, who had initially successfully quit smoking under Bupropion SR (Zyban ${ }^{\mathrm{TM}}$ ), were again treated with Bupropion SR for smoking cessation. The encouraging results prompted us to perform a study in Germany under the conditions and temporal restraints of a routine medical practice. Methods/Patients: In this open multicentre study 321 patients of a total of 365 patients at 62 study sites were included. The patients represented a healthy population ( = free of severe chronic disease) of smokers, who had relapsed after a smoking cessation attempt with Bupropion SR or nicotine replacement therapy and were currently smoking at least $20 \mathrm{cig}$./d for $>3$ months. The treatment with Bupropion SR (300 mg/d) lasted 7 weeks, with initial run-in of $150 \mathrm{mg} / \mathrm{d}$ for the first three days; motivational counselling was provided during 4 clinical visits within the study duration of 26 weeks. Results: $55.4 \%$ of the patients were heavily or very heavily nicotine dependent as detected by the Fagerström Tolerance Questionnaire (score 6-11). The continuous abstinence rates for week $4-7$ were $29.6 \%$ (ITT) the point prevalence rates for week 26 were $30.5 \%$ (ITT). Patients who showed a relapse had less daily cigarette consumption even after 26 weeks (10.3 cig./d versus $26.9 \mathrm{cig}$./d pretreatment). Nicotine craving reached a low plateau not earlier than week 6 . Bupropion SR was well tolerated, only $9.7 \%$ of the patients (ITT) 
Schlafstörungen. Schlussfolgerung: Bupropion SR erwies sich als gut wirksam und verträglich in der Raucherentwöhnung bei rückfälligen Rauchern. withdrew from the study due to an adverse event. The most frequent adverse events were pain of the musculoskeletal system and insomnia. Conclusions: Bupropion SR was effective and well tolerated when given as an aid in smoking cessation in relapsed smokers.

\section{Einleitung}

Bei der Nikotinabhängigkeit handelt es sich um eine Suchterkrankung. Dies erklärt die sehr niedrigen Erfolgsraten nach 1 Jahr, wenn der Patient die Entwöhnung weitgehend ohne medikamentöse oder anderweitig therapeutische Unterstützung vornimmt: Selbst unter Studienbedingungen, in denen die Kontrollund Verumgruppe regelmäßig kontaktiert wurden, waren die Abstinenzraten in der Plazebogruppe mit 7,9\% und in der Verumgruppe (hausärztliche Unterweisung ohne medikamentöse oder verhaltenstherapeutische Unterstützung) mit nur 10,2\% gering ([1], S. 57).

Sowohl US-amerikanische [2] als auch deutsche Therapierichtlinien [3] empfehlen daher prinzipiell neben beratenden/verhaltenstherapeutischen Maßnahmen eine pharmakotherapeutische Unterstützung bei der Raucherentwöhnung (Nikotinersatzpräparate, Bupropion SR).

Bupropionhydrochlorid SR (Slow Release, Zyban ${ }^{\circledR}$ ) hat sich in klinischen Prüfungen als ein wirksames, nikotinfreies Medikament in der Raucherentwöhnung erwiesen, wenn es als Bestandteil eines Raucherentwöhnungsprogrammes bei Rauchern eingesetzt wird, die motiviert sind, das Rauchen aufzugeben. Bupropion SR ist seit Juni 2000 in Deutschland als verschreibungspflichtiges Medikament für die Indikation Raucherentwöhnung zugelassen.

Der Wirkmechanismus von Bupropion SR bei der Entwöhnung des Rauchens ist noch nicht endgültig geklärt. Als Noradrenalinund Dopamin-Wiederaufnahmehemmer führt Bupropion SR analog den Wirkungen von Nikotin - zu einer Erhöhung der entsprechenden Neurotransmitter-Konzentrationen in bestimmten Hirngebieten (u.a. Nucleus accumbens und Locus coeruleus), und soll so Entzugssymptome und Rauchdrang vermeiden. Dagegen weist Bupropion SR selbst kein erhöhtes Abhängigkeitspotenzial auf [4].

In zwei multizentrischen, randomisierten, doppelblinden und plazebokontrollierten Studien mit 613 (Dosisfindungsstudie) bzw. 893 Teilnehmern (Vergleichsstudie gegen Plazebo und Nikotinpflaster) wurde Bupropion SR $300 \mathrm{mg}$ (2-mal täglich $150 \mathrm{mg}$ ) über eine Behandlungszeit von 7 bzw. 9 Wochen angewendet und erwies sich gegenüber Plazebo und in der zweiten Studie auch gegenüber Nikotinpflaster als signifikant überlegen. Der Therapieeffekt war sowohl kurzfristig als auch langfristig nachweisbar: Die kontinuierlichen Abstinenzraten von Woche 4 bis 7 betrugen in der Dosisfindungsstudie 36\% (BUP SR) vs. $17 \%$ (Plazebo) und in der Vergleichsstudie 49\% (BUP SR) vs. 36\% (Nikotinpflaster) vs. $23 \%$ (Plazebo). Die Punktprävalenzraten nach 12 Monaten waren in der Dosisfindungsstudie 23,1\% (Bupropion
SR) vs. 12,4\% (Plazebo) und in der Vergleichsstudie 30,3 (Bupropion SR) vs. 16,4\% (Nikotinpflaster) vs. 15,6\% (Plazebo) [5,6].

Durch den Suchtcharakter der Nikotinabhängigkeit gehören wiederholte Rückfälle zum klinischen Bild: Ein erfolgreich entwöhnter Ex-Raucher hat durchschnittlich 6 Entwöhnungsversuche durchgemacht [1]. Nach einem misslungenen Raucherentwöhnungsversuch verschlechtern sich die Erfolgsquoten für die nächsten Abstinenzversuche weiter: Bei wiederholtem Einsatz von Nikotinersatztherapie sank die Erfolgsrate in drei bisher zu diesem Thema durchgeführten Studien mit Nikotinpflaster $[7,8]$ und dem Nikotin-Inhaler [9] beim zweiten Entwöhnungsversuch auf $0-6,4 \%$ nach 6 Monaten.

In der vorliegenden Studie sollten Wirksamkeit und Verträglichkeit von Bupropion SR $(2 \times 150 \mathrm{mg} / \mathrm{Tag})$ in der Raucherentwöhnung nach einem misslungenen Entwöhnungsversuch mit Bupropion SR oder Nikotinersatztherapie geprüft werden. Ein offenes Design wurde gewählt, um möglichst genau die Bedingungen und zeitlichen Limitierungen des Praxisalltags widerzuspiegeln.

\section{Patienten und Methoden}

In diese multizentrische, offene, einarmige und nicht-randomisierte Studie bei niedergelassenen Allgemeinärzten, Internisten und Pneumologen wurden Raucher ab 18 Jahren eingeschlossen, die seit mindestens 5 Jahren 20 Zigaretten pro Tag oder mehr rauchten. Sie mussten dabei nicht primär wegen des Wunsches nach einer Raucherentwöhnung den Arzt aufgesucht haben, der Grund des Arztbesuches wurde nicht dokumentiert. Sie mussten in dem der Studie vorausgehenden Jahr einen gescheiterten Entwöhnungsversuch unter Bupropion SR oder Nikotinersatztherapeutika (Pflaster, Kaugummi, Spray, Inhaler) unternommen haben, wobei sie zumindest 1 Tag vollständig abstinent gewesen sein mussten, um die Ernsthaftigkeit des Entwöhnungsversuches zu dokumentieren. Sie mussten motiviert für eine Entzugsbehandlung sein. Ausschlusskriterien waren u.a. epileptische Anfälle in der Anamnese, bekannte Risikofaktoren für epileptische Anfälle, relevante internistische Vorerkrankungen oder eine Anamnese einer bipolaren Psychose oder Essstörung.

Die Studie teilte sich in eine 7-wöchige Behandlungsphase und eine 19-wöchige Nachbeobachtungsphase. In der Screening-Visite wurden die Ein- und Ausschlusskriterien geprüft, der Fagerström-Test zur Ermittlung des Grades der Nikotinabhängigkeit durchgeführt, eine vollständige Anamnese einschließlich der Begleitmedikation erhoben und eine körperliche Untersuchung einschließlich der Vitalwerte und dem Körpergewicht durchgeführt. Ferner wurde ein EKG angefertigt und ein Routinelabor er- 
RVT= Rauchverzichtstag (individuell festzulegen für Woche 2)

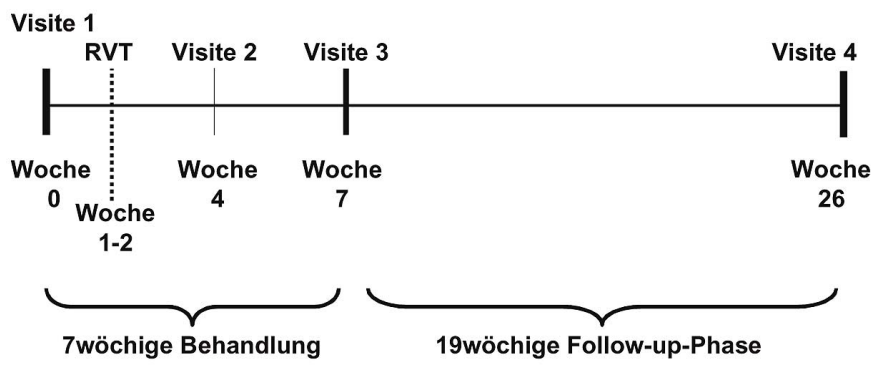

Abb. 1 Studiendesign.

hoben und bei Frauen im gebärfähigen Alter zusätzlich ein Schwangerschaftstest durchgeführt.

Nach Vorliegen der Einverständniserklärung wurde mit der Gabe von Bupropion SR nach dem damals gültigen Dosierungsschema begonnen (in den ersten 3 Tagen $1 \times 150 \mathrm{mg} / \mathrm{Tag}$, ab Tag 4 $2 \times 150 \mathrm{mg} /$ Tag für insgesamt 7 Wochen). Der so genannte Rauchverzichtstag (RVT) wurde individuell als der Tag in der 2. Behandlungswoche vereinbart, an dem der Studienteilnehmer schlagartig und endgültig mit dem Rauchen aufhören sollte (Abb.1). Am Tag vor dem RVT erfolgte ein motivierender Telefonanruf durch den Studienarzt. Bei den beiden für die Behandlungsphase vorgesehenen Visiten in Woche 4 und 7 sowie der Studienabschlussvisite in Woche 26 wurde neben der Erhebung des Raucherstatus, einer körperlichen Untersuchung und der Dokumentation etwaiger unerwünschter Ereignisse ein kurzes (<10 min) strukturiertes Beratungs- und Motivationsgespräch nach P. Hajek (Psychology Section, Queen Mary's School of Medicine and Dentistry, London) geführt. Initial und mehrfach im Verlauf wurde zudem die Stimmungslage mit dem Beck-Depressions-Inventar erhoben.

Während der 7-wöchigen Therapiephase wurde täglich einmal zu einer festgesetzten Zeit (früher Abend) die Einnahme der Studienmedikation, die Zahl der jeweils gerauchten Zigaretten sowie das subjektive Verlangen nach einer Zigarette („Craving“, Skala von 0-3) mit einem elektronischen Patiententagebuch (Symfo ${ }^{\circledR}$ Electronic Diary, Firma Symfo SA, Brüssel) erfasst, um realitätsnähere Angaben über den Zigarettenkonsum zu gewinnen (in der 19-wöchigen Follow-up-Phase wurde ein nicht-elektronisches wöchentliches Tagebuch geführt).

Der primäre Endpunkt der Studie war die kontinuierliche Abstinenzrate von Woche 4-7 (= Tag 22-49) der Behandlung, d.h. die Angabe des Patienten in seinem elektronischen Tagebuch, im Beobachtungszeitraum keine einzige Zigarette geraucht $\mathrm{zu}$ haben. Fehlten auf dem elektronischen Tagebuch die Angaben zum Zigarettenkonsum an 3 aufeinanderfolgenden Tagen, so wurde Rückfälligkeit angenommen. Dies hatte zur Folge, dass ein Patient, obwohl niemals das Rauchen einer Zigarette dokumentiert wurde, als rückfälliger Raucher gezählt wurde.

Sekundäre Endpunkte waren kontinuierliche Abstinenzraten von Woche 4-26, von RVT bis Woche 4, 7 und 26, die Punktprävalenzraten (d.h. die Angabe, in der Woche vor der Erhebung keine einzige Zigarette geraucht zu haben) in den Wochen 7 und 26, der mittlere Zigarettenkonsum in Woche 4, 7 und 26, unerwünschte Wirkungen, Craving, Stimmung und Körpergewicht.

Die Hauptzielgröße der Studie wurde für ein „Intent-totreat“-Kollektiv ausgewertet, zum ebenfalls ausgewerteten Perprotocol-Kollektiv gehörten dagegen alle Patienten, bei denen keinerlei gravierende Verstöße gegen den Prüfplan festgestellt und die bis zum Besuch 4 dokumentiert wurden. Bei fehlenden Eintragungen/Angaben des Probanden wurde eine Rückfälligkeit angenommen.

Die Studie wurde durch die lokal zuständigen Ethik-Kommissionen genehmigt und in Übereinstimmung mit den Richtlinien des „Good Clinical Practice“ durchgeführt.

\section{Ergebnisse}

\section{Wirksamkeit}

In insgesamt 62 Studienzentren (niedergelassene Allgemeinärzte, Internisten und Pneumologen) wurden 365 Patienten in die Studie eingeschlossen und nahmen Bupropion SR wenigstens einmal ein (Population zur Analyse der Verträglichkeit). Bei 44 Patienten lagen aber nur keine oder sehr lückenhafte Daten vor, so dass 321 Patienten in die Intent-to-treat-Population zur Auswertung der Wirksamkeit kamen. 172 Patienten durchliefen die gesamte Studie ohne gravierende Prüfplanverstöße, diese bildeten die Per-Protocol-Gruppe. In den Tab. 1 und $\mathbf{2}$ sind die allgemeine Demografie und die Raucher- und Entwöhnungsanamnese zusammengefasst; Abb. 2 gibt die Verteilung der Patienten auf die Grade der Nikotinabhängigkeit nach dem Fagerström Tolerance Questionnaire wieder; 55,4\% der Patienten waren stark oder sehr stark nikotinabhängig.

Tab. 1 Demografie der ITT-Population

\begin{tabular}{|c|c|c|c|}
\hline & gesamt & Männer & Frauen \\
\hline Studienpatienten (n) & 321 & 170 & 151 \\
\hline $\begin{array}{l}\text { Durchschnittsalter } \\
\text { (Jahre } \pm \text { Standardabweichung) }\end{array}$ & $43,3 \pm 11,2$ & $44,4 \pm 11,2$ & $42,1 \pm 11,0$ \\
\hline $\begin{array}{l}\text { Körpergewicht } \\
\text { (kg } \pm \text { Standardabweichung) }\end{array}$ & $75,7 \pm 15,2$ & $82,8 \pm 12,2$ & $67,6 \pm 14,1$ \\
\hline
\end{tabular}

Tab. 2 Raucher- und Entwöhnungsanamnese

\begin{tabular}{ll}
\hline täglicher Zigarettenkonsum (n) & $26,9 \pm 8,9$ \\
\hline Raucheranamnese (Jahre) & $21,7 \pm 10,4$ \\
\hline Zahl der durchgemachten Entwöhnungsversuche (n) & $3,4 \pm 2,8$ \\
früherer Entwöhnungsversuch mit (in \% der Studien- & \\
patienten, mehr als eine Nennung möglich): & \\
- Nikotinpflaster & 44,5 \\
- Nikotinkaugummi & 34,9 \\
- Bupropion SR & 19,0 \\
- Akupunktur & 2,3 \\
- Bücher & 0,7 \\
- psychologische Unterstützung & 0,7
\end{tabular}




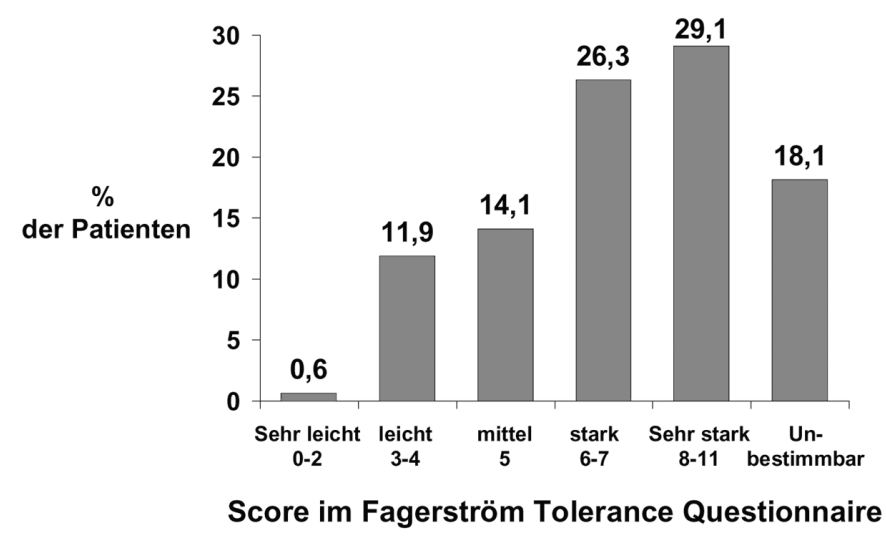

Abb. 2 Prozentuale Verteilung des Grades der Nikotinabhängigkeit der Studienpatienten, gemessen mit dem Fagerström Tolerance Questionnaire.

Sämtliche im Folgenden genannten Abstinenzraten beziehen sich auf die ITT-Population, die Abstinenzraten der Per-ProtocolPopulation sind in Klammern kursiv gesetzt:

Die kontinuierliche Abstinenzrate von Woche 4-7 bzw. Tag $22-49$ (prim. Endpunkt) betrug 29,6\% (95\%-Konfidenzintervall: 24,6 - 34,6\%; 38,4\%), beinahe ein Drittel der Patienten waren also in der zweiten Hälfte der Therapiephase mit Bupropion SR vollständig rauchfrei. In der Subgruppe der Patienten mit einem vorangegangenen Entwöhnungsversuch unter Bupropion SR lag die kontinuierliche Abstinenzrate für Woche $4-7$ bei $34,4 \%$ (21 von 61 Pat.), bei der Subgruppe mit vorangegangenem Entwöhnungsversuch unter Nikotinersatztherapie betrug sie 28,5\% (74 von 260). Dieser Unterschied war nicht signifikant.

Die langfristige kontinuierliche Abstinenzrate von Woche 4-26 betrug 15,3\% (20,3\%), d.h. etwas mehr als die Hälfte der nach 7 Wochen abstinenten Patienten $(51,6 \%=49$ von 95 Patienten) blieben bis zum Abschluss der Studie nach 26 Wochen rauchfrei. Die langfristige kontinuierliche Abstinenzrate vom Rauchverzichtstag bis Woche 26 betrug 11,5\% (15,7\%). Wenn das weniger strenge Maß der Punktprävalenzrate nach 26 Wochen angewendet wird, ergibt sich eine Abstinenzrate von 30,5\% (40,7\%).

Nach Abschluss der Studie beurteilten 75,2\% der Studienärzte und 72,2\% der Patienten die Wirksamkeit von Bupropion SR als „gut" oder „sehr gut“.

Es fällt auf, dass die rückfälligen Raucher auch nach ihrem Rückfall einen deutlich geringeren täglichen Zigarettenkonsum als vor der Behandlung mit Bupropion SR aufweisen (Abb. 4). Dieser Effekt ist langfristig nachweisbar: Auch nach 26 Wochen liegt der Konsum bei durchschnittlich 10,3 (zuvor 26,9) Zigaretten täglich (Datenbasis bei der letzten Visite: 121 rückfällige Raucher).

Das Craving nach einer Zigarette nahm bei den abstinenten Rauchern in den ersten Wochen deutlich ab und erreichte in Woche 6 ein niedriges Plateau, das im weiteren Verlauf bis zum Abschluss der Studie nicht unterschritten wurde (Abb.5). Auch nach planmäßigem Absetzen von Bupropion SR nach der 7. Therapiewoche nahm das Craving nicht mehr zu.
$11,5 \%$

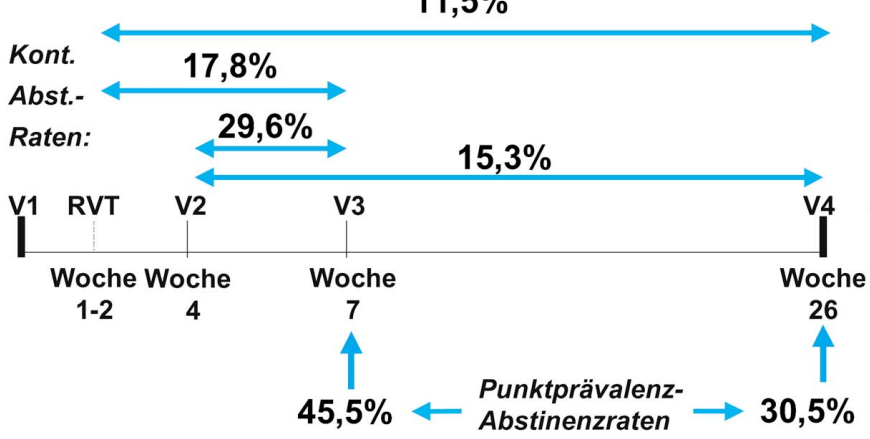

Abb. 3 Kontinuierliche und Punktprävalenz-Abstinenzraten der Intent-to-treat-Population (V1-4 = Visiten 1-4, RVT = Rauchverzichtstag) (blaue Pfeile bezeichnen die Punktprävalenz, die horizontalen blauen Pfeile bezeichnen die kontinuierlichen Abstinenzraten zwischen zwei Zeitpunkten)

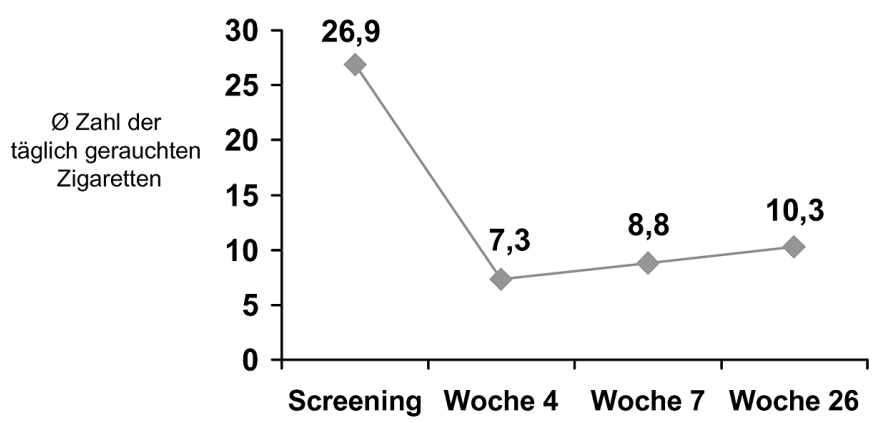

Abb. 4 Entwicklung des durchschnittlichen täglichen Zigarettenkonsums der rückfälligen Raucher (ITT-Population).

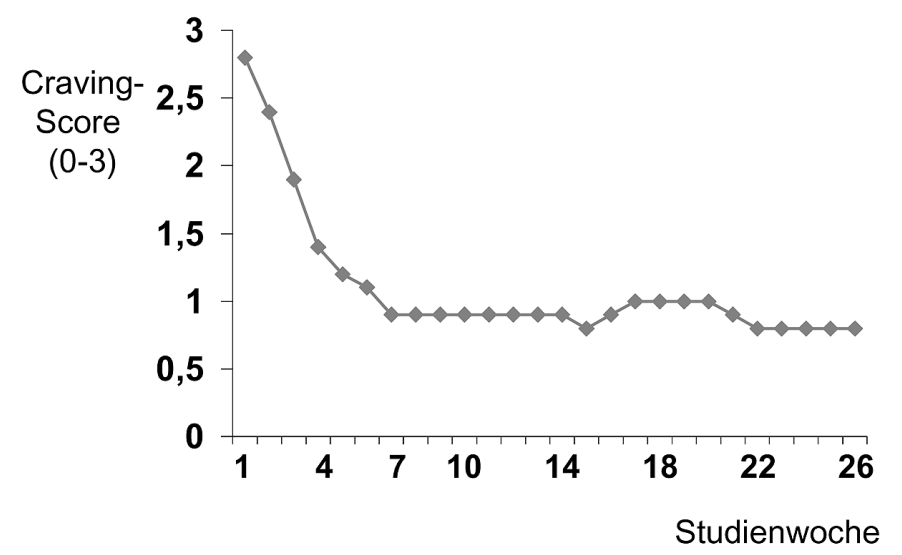

Abb. 5 Entwicklung des Cravings über die Studiendauer bei den kontinuierlich von Woche 4-7 abstinenten Rauchern (erhoben in der 7-wöchigen Therapiephase täglich mittels elektronischem Tagebuch, in der Nachbeobachtungsphase mittels wöchentlichem Papiertagebuch).

Der Gesamtscore des Beck-Depressions-Inventars fiel während der Therapiephase von 4,9 $\pm 5,7$ auf 2,7 $\pm 4,5$. Hierbei fielen bei den Patienten, die von Woche 4-7 abstinent waren, die Werte etwas stärker $(5,1->2,6)$ als bei den in diesem Zeitraum nicht abstinenten Patienten (4,7-> 2,7). 


\section{Verträglichkeit}

197 von 365 initial eingeschlossenen Patienten (54\%) berichteten von mindestens einem unerwünschten Ereignis, 36\% aller unerwünschten Ereignisse wurden hierbei durch den Studienarzt als möglicherweise, wahrscheinlich oder sicher durch Bupropion SR verursacht gewertet. In Tab. 3 sind die häufigsten unerwünschten Ereignisse aufgeführt. Bei 31 der 197 Patienten $(15,7 \%)$ führte ein unerwünschtes Ereignis zum Therapieabbruch, dies entspricht 9,7\% der Intent-to-treat-Population.

Insgesamt wurde bei 8 Patienten über das Auftreten von schweren unerwünschten Ereignissen berichtet. Bei 2 Patienten standen diese nach Meinung der Ärzte in einem Zusammenhang mit der Einnahme mit Bupropion SR: Eine 34-jährige Frau erlitt einen Grand-mal-Anfall (wahrscheinlicher Zusammenhang), ein 20-jähriger Mann erlitt eine Yersinia-enterocolitica-Infektion mit reaktiver Urticaria, Bronchialasthma sowie Arthritis beider Hände, Schultern und Sternoklavikulargelenke (als „möglicher Zusammenhang“ vom Prüfarzt gewertet). Beide Ereignisse bildeten sich nach Absetzen der Studienmedikation unter spezifischer Therapie vollständig zurück.

Während der 7-wöchigen Therapie betrug die durchschnittliche Gewichtszunahme in der ITT-Population 1,6 kg (Frauen: 1,6 kg, Männer: $0,9 \mathrm{~kg}$ ). Vom Ende der Therapie bis zum Ende der 19-wöchigen Nachbeobachtungsphase betrug die durchschnittliche Gewichtszunahme wiederum 1,6 kg (Frauen: 1,6 kg, Männer: $1,9 \mathrm{~kg}$ ).

Unter der Behandlung mit Bupropion SR (von Visiten 1 - 3) nahm der mittlere systolische Blutdruck von $126,5 \pm 14,9 \mathrm{~mm} \mathrm{Hg}$ auf $127,8 \pm 15,9 \mathrm{~mm} \mathrm{Hg}$ und der diastolische Blutdruck von $78,7 \pm$ $8,9 \mathrm{~mm} \mathrm{Hg}$ auf $79,0 \pm 7,8 \mathrm{~mm} \mathrm{Hg}$ zu (ITT-Population). Von allen 321 Patienten, die in der ITT-Population waren und somit mindestens einmal Studienmedikation erhalten hatten, trat bei $6 \mathrm{~Pa}-$ tienten eine Hypertonie neu auf ( und führte bei einem dieser Patienten zum Absetzen von Bupropion SR), bei einem Patienten verschlechterte sich eine vorbestehende Hypertonie.

\section{Diskussion}

Gemäß des Suchtcharakters der Nikotinabhängigkeit sind Rückfälle nach einer Raucherentwöhnung sehr häufig und stellen wegen der mit der Zahl der erfolglosen Versuche steigenden Frustration bei Patient und Arzt eine große therapeutische Herausforderung dar. Auch unter wiederholter medikamentöser Unterstützung in Form von Nikotinersatztherapie werden eher geringe Erfolgsraten bei rückfälligen Rauchern berichtet [7-9].

Diese offene, nicht-randomisierte, einarmige Studie über 26 Wochen bei 62 Allgemeinärzten, Internisten und Pneumologen in Deutschland untersuchte die Wirksamkeit und Verträglichkeit von Bupropion SR in der ambulanten Raucherentwöhnung bei entwöhnungsmotivierten Patienten, die im der Studie vorausgegangenen Jahr einen erfolglosen medikamentös gestützten Entwöhnungsversuch durchgemacht hatten. Das Design der Studie mit insgesamt 4 Visiten und motivierenden Beratungsgesprächen war an die vielerorts maximal möglichen Ressourcen und die Therapiemöglichkeiten der ambulanten Raucherentwöh-
Tab. 3 Häufigste unerwünschte Ereignisse (bezogen auf 365 Patienten, die mindestens einmal Studienmedikation einnahmen)

\begin{tabular}{|ll}
\hline Art des unerwünschten Ereignisses & Häufigkeit (\%) \\
\hline Schmerzen des Bewegungsapparates & 11,0 \\
\hline Schlafstörungen & 9,6 \\
\hline Bronchitis/COPD & 6,6 \\
\hline Gastritis & 5,5 \\
\hline Infektion & 5,2 \\
\hline Verdauungsstörung/Verstopfung/Diarrhö & 4,9 \\
\hline Gewichtszunahme & 4,7 \\
\hline Schwindel & 4,1 \\
\hline Kopfschmerzen & 4,1 \\
\hline Übelkeit/Erbrechen & 3,8 \\
\hline Grippe-ähnliche Symptome & 3,6 \\
\hline Bauchschmerzen & 3,0 \\
\hline (Verschlechterung einer) Hypertonie & 2,7 \\
\hline Allergie/Urticaria & 2,5 \\
\hline
\end{tabular}

nung in der Primärversorgung angelehnt. Diese Studie mit ihrem geringeren Aufwand bildete somit den Therapiealltag in der deutschen primärärztlichen Versorgung vermutlich besser ab als die meisten internationalen Raucherentwöhnungsstudien (mit z.T. wöchentlichen Visiten).

Das während der 7-wöchigen Therapiephase verwendete elektronische Tagebuch erinnnerte die Patienten einerseits einmal täglich an die Medikamenteneinnahme und erhob andererseits zeitnah die Anzahl der täglich gerauchten Zigaretten sowie das Craving nach einer Zigarette. Die erzwungenermaßen tägliche Abfrage des Cravings generiert realitätsnähere Daten als das retrospektive Ausfüllen von Papiertagebüchern, wie in Studien oft üblich. Da Craving eine psychophysiologische Momentaufnahme darstellt und über die Dauer eines Tages stark fluktuieren kann, wäre es zwar wünschenswert gewesen, eine noch engmaschigere als $1 \times$ tägliche Erhebung durchzuführen. Dies hätte allerdings erfordert, dass der Patient permanent das Instrument mitgeführt hätte, was die Compliance der Studienteilnahme vermutlich deutlich gesenkt hätte. Außerdem war die Verwendung dieses elektronischen Patiententagebuches auch zur Erhöhung der Patientencompliance vorgesehen; dieser potenzielle Effekt kann jedoch nicht gemessen werden.

Das Patientenkollektiv war typisch für nationale und internationale Interventionsstudien in der Raucherentwöhnung: Eine Raucheranamnese von im Mittel mehr als 20 Jahren, bereits mehrere durchgemachte Entwöhnungsversuche und $\mathrm{zu}$ mehr als der Hälfte starke und sehr starke Nikotinabhängigkeit (FagerströmTest) belegen eine hochgradig abhängige und damit therapiebedürftige Patientengruppe, die ohne psychologische und/oder medikamentöse Unterstützung kaum langfristig rauchfrei wird. In den vorangegangenen Entwöhnungsversuchen hatten beinahe 80\% der Patienten Erfahrungen mit Nikotinersatztherapie gemacht, aufgrund der in Deutschland zum Zeitpunkt der Studie erst etwa seit $1 \mathrm{Jahr}$ bestehenden Zulassung nur 19\% mit Bupropion SR. 
Bei der Beschreibung der Wirksamkeit einer Raucherentwöhnungstherapie sind international zwei Maße gebräuchlich: Die kontinuierliche Abstinenzrate über einen bestimmten Zeitraum (z.B. Woche 4-7, Woche 4-26) stellt einen besonders strengen Maßstab der Abstinenz dar, sie erlaubt keinerlei „Ausrutscher“ oder Rückfälle. Die realitätsnähere Punktprävalenzrate dagegen macht eine Aussage über die Abstinenz in einer bestimmten Studienwoche oder einem Studienmonat (z.B. Monat 6), erlaubt daher (in engen Grenzen) „Ausrutscher“, ohne diese sofort als Therapieversager zu werten. Entsprechend sind daher die Punktprävalenzabstinenzraten meist höher als die kontinuierlichen Abstinenzraten.

Bupropion SR erwies sich in dieser Studie als wirksam in der Unterstützung der Raucherentwöhnung: Fast ein Drittel der Patienten $(29,6 \%)$ war während der letzten 4 Wochen der Therapiephase vollständig rauchfrei, waren also in der initialen Entwöhnung erfolgreich, die ohne medikamentöse Unterstützung durch besonders große Rückfallgefahr gekennzeichnet ist. Nach Hughes [10] werden ohne medikamentöse Unterstützung bereits in der ersten Woche nach Rauchstopp 65\% der entwöhnungswilligen Patienten wieder rückfällig. Dies zeigt, dass Bupropion SR die Symptome der neurobiologischen Entgiftung in den ersten Wochen bei vielen Rauchern mildert.

Angesichts der hohen Abhängigkeitsrate der Patienten sind die langfristigen Abstinenzraten von besonderem Interesse: Die Hälfte der initial erfolgreichen Patienten verblieb auch bis zum Ende der Studie komplett rauchfrei (kontinuierliche Abstinenz Woche 4-26:15,3\%), während in der weniger strengen, gleichwohl realitätsnäheren Punktprävalenzabstinenzrate in der 26. Woche noch 30,5\% der Patienten Rauchfreiheit angaben.

Kritisch ist anzumerken, dass keine Überprüfung der Angaben des Patienten z.B. durch eine Messung der CO-Konzentration in der Ausatemluft oder eine Cotinin-Bestimmung im Urin erfolgte, die ein semiquantitatives Maß der in den letzten 24 Stunden bzw. 3 Tagen gerauchten Zigaretten darstellen. Angesichts des multizentrischen offenen Charakters und der engmaschigen Erhebung des Rauchstatus während der Therapiephase durch ein elektronisches Tagebuch erschien diese Verifizierung als verzichtbar.

Es zeigte sich kein signifikanter Unterschied des Entwöhnungserfolges in Abhängigkeit des beim letzten Abstinenzversuch verwendeten Medikamentes; die leicht höhere Abstinenzrate für Bupropion SR (34,4\% versus $28,5 \%$ für Nikotinersatztherapeutika) kann zum einen durch die größere Streuung der Rate aufgrund der deutlich kleineren Fallzahl der mit Bupropion vorbehandelten Gruppe erklärt werden. Zum anderen kann aber auch ein Bias vorliegen, da diese Patienten bei ihrem vorangegangenen Entwöhnungsversuch Bupropion SR gut vertragen hatten und somit in der vorliegenden Studie ein Therapieabbruch durch schlechte Verträglichkeit seltener auftrat.

Interessanterweise hielt bei den rückfälligen Rauchern auch nach der gesamten Studiendauer noch die Reduktion des täglichen Zigarettenkonsums an (von 26,9 auf 10,3), wenn auch die Datenbasis bei dieser Patientengruppe nach 26 Wochen geringer war (121 rückfällige Raucher), so dass zumindest diesbezüglich auch ein Benefit für rückfällige Raucher zu vermuten ist.

Vor dem Hintergrund des offenen Charakters der Studie ist ein Vergleich mit plazebokontrollierten Studien schwierig: Gonzales u. Mitarb. [11] fanden in einer multizentrischen, randomisierten, doppelblinden und plazebokontrollierten Studie mit einem zuvor gescheiterten Entwöhnungsversuch mit Bupropion SR bei einem erneuten 12-wöchigen Therapieversuch mit Bupropion SR hochsignifikant bessere Entwöhnungsraten unter Bupropion SR als unter Plazebo auch noch nach 26 Wochen (kont. Abstinenzrate 12 vs. $2 \%$, p <0,001). Zu dieser deutlichen Überlegenheit von Bupropion SR mag beigetragen haben, dass die Patienten sämtlich Erfahrung mit Bupropion SR hatten und das Plazebomedikament als solches erkennen konnten.

Da das von den Patienten berichtete Craving erst in der 6. Woche ein niedriges Plateau erreichte, kann der Patient bei zu frühem Absetzen unnötig durch starkes Craving in große Rückfallgefahr geraten. Eine hinreichend lange Therapiedauer von 7-9 Wochen ist außerdem wie auch bei anderen Raucherentwöhnungstherapien deshalb notwendig, da der Raucher erst nach gewisser Zeit beginnt, seine typischen Raucher-Verhaltensweisen abzulegen, d. h. alternative Strategien zur Stressbewältigung oder Beschäftigung der Hände zu entwickeln.

Bei einer Raucherentwöhnung kann es durch den Entzug des antidepressiv wirksamen Nikotins zu leichten, aber auch schweren Depressionen kommen [15]. In der vorliegenden Studie zeigte der durchschnittliche Score des Beck Depressions-Inventares eine euthyme Studienpopulation; die geringe durchschnittliche Abnahme nach Abschluss der Therapiephase kann als klinisch nicht relevant angesehen werden. Wegen der zudem fehlenden Plazebokontrolle kann in der vorliegenden Studie keine Aussage über eine möglicherweise günstige Modulation der Stimmung nachgewiesen werden.

Bei der Analyse der unerwünschten Ereignisse ist zu beachten, dass die Raucherentwöhnung selbst einen Entgiftungsprozess von der psychotropen Substanz Nikotin darstellt und daher insbesondere mit zentralnervösen Begleiterscheinungen zu rechnen ist. Durch den unkontrollierten Charakter der Studie können diese unerwünschten Wirkungen der Nikotinentwöhnung nicht von den medikamenten-induzierten unerwünschten Wirkungen differenziert werden. Aus plazebo-kontrollierten Studien ist bekannt, dass insbesondere Schwindel, Übelkeit/Erbrechen, Verdauungsstörungen, Kopfschmerzen, Konzentrations- und Schlafstörungen auch in der Plazebogruppe als Ausdruck der Nikotinentwöhnung auftreten [5,6]. Die unter Bupropion SR bekanntermaßen gegenüber Plazebo vermehrt beobachteten Schlafstörungen traten in der vorliegenden Studie mit unter $10 \%$ seltener als in den plazebokontrollierten Studien auf, vermutlich weil die Patienten vom Arzt Strategien zur Vermeidung erhielten (Einnahme der zweiten Tagesdosis relativ früh am Tag).

Eine von 365 eingeschlossenen Patienten erlitt einen epileptischen Anfall, ohne dass Risikofaktoren zu eruieren gewesen wären. Für Bupropion SR ist (bei depressiven Patienten) ein Risiko von $0,1 \%$ für das Auftreten epileptischer Anfälle beschrieben [12]. Daher stellt eine Anamnese jeglicher Krampfanfälle eine 
Kontraindikation dar; bei Vorliegen von Risikofaktoren wie Zustand nach schwerem Schädel-Hirn-Trauma oder Schlaganfall muss eine sorgfältige Nutzen-Risiko-Abwägung erfolgen.

Ein Patient erlitt eine Yersinia-enterocolitica-Infektion, die vom Prüfarzt als möglicherweise durch Bupropion SR verursacht eingeschätzt wurde; der Grund für die Annahme einer möglichen Kausalität blieb unklar.

Durch eine Raucherentwöhnung wird der Grundumsatz reduziert bzw. normalisiert, woraus oftmals eine Gewichtszunahme resultiert, die die Compliance vor allem von Patientinnen verringert. In der vorliegenden Studie nahmen die Patienten durchschnittlich moderat zu, in der 7-wöchigen Therapiephase etwa gleichviel wie in der 19-wöchigen Nachbeobachtungsphase, wobei Frauen zumindest in der Therapiephase eine größere Gewichtszunahme aufwiesen. 4,7\% der Patienten berichteten eine Gewichtszunahme. Durch das offene Design der Studie können keine Aussagen über einen möglichen günstigen Effekt von Bupropion SR auf die Gewichtszunahme getroffen werden; in den randomisierten, kontrollierten und doppelblinden Studien war unter Bupropion SR während der Therapiephase eine geringere durchschnittliche Gewichtszunahme als unter Plazebo beobachtet worden. Dieser Unterschied verlor sich allerdings nach Ende der Therapie mit Bupropion SR.

In der vorliegenden Studie veränderte sich der mittlere systolische und diastolische Blutdruck klinisch nicht signifikant. Jüngst wurde in einer randomisierten internationalen Studie bei Patienten mit kardiovaskulären Vorerkrankungen ebenfalls kein klinisch signifikanter Effekt von Bupropion SR auf den arteriellen Blutdruck und die Herzfrequenz im Vergleich zu Plazebo gefunden; zur Neumanifestation bzw. Verschlechterung eines vorbestehenden Hypertonus kam es bei 2 von 313 Patienten in der Bupropion-SR-Gruppe und bei 3 von 313 Patienten in der Plazebogruppe [13]. Auch in der vorliegenden Studie wurden einzelne Neumanifestationen oder Verschlechterungen einer vorbestehenden Hypertonie beobachtet, eine gelegentlich auftretende Komplikation. In einer kontrollierten Studie traten Blutdruckerhöhungen bei der Kombination von Bupropion SR mit einem Nikotinpflaster signifikant häufiger als unter Bupropion SR alleine auf, weswegen bei der kombinierten Gabe wöchentliche Blutdruckkontrollen empfohlen werden [6].

In der vorliegenden Studie brach nur jeder zehnte der Patienten aus der Intent-to-treat-Population die Studie aufgrund unerwünschter Ereignisse ab. Der obengenannte Bias in der Subgruppe der mit Bupropion SR vorbehandelten Patienten tritt allerdings auch bei der Auswertung der Verträglichkeit möglicherweise in abgeschwächter Form auf, da zumindest die geringe Zahl der mit Bupropion SR vorbehandelten Patienten beim ersten Therapieversuch keine schwerwiegenden Unverträglichkeiten erlitten haben durften.

Zur Prävention aber auch Therapie von pneumologischen Raucherfolgeerkrankungen ist eine Raucherentwöhnung von entscheidender Bedeutung. Tashkin u. Mitarb. [14] fanden in einer doppelblinden randomisierten und plazebokontrollierten Studie bei COPD-Patienten eine signifikante Überlegenheit von Bupropion SR gegenüber Plazebo. Angesichts der starken Abhängigkeit der Patienten und der langen Raucheranamnese (durchschnittlich 51 „Päckchenjahre“, einem international gebräuchlichen $\mathrm{Ma}$ der kumulativen lebenslangen Tabakexposition, definiert als Produkt der täglich gerauchten Packung Zigaretten à 20 Zigaretten und der Dauer des Tabakkonsums in Jahren) waren die absoluten Abstinenzraten sowohl unter Bupropion SR als auch unter Plazebo niedriger als in vergleichbaren Studien (kont. Abstinenzrate Woche $4-728 \%$ vs. $16 \%, p=0,003$; kont. Abstinenzrate Woche $4-2616$ vs. $9 \%, p=0,04)$.

Zusammenfassend erwies sich Bupropion SR als wirksam und gut verträglich zur Unterstützung einer erneuten Raucherentwöhnung bei Patienten, die bereits mindestens einen gescheiterten medikamentös unterstützten Entwöhnungsversuch durchgemacht hatten.

Sponsor dieser Studie war GlaxoSmithKline, München.

\section{Literatur}

${ }^{1}$ Fiore MC, Bailey WC, Cohen SJ et al. Treating tobacco use and dependence. Clinical Practice Guideline. Rockville (MD): US Department of Health and Human Services, Public Health Service, June 2000

2 The Tobacco Use and Dependence Clinical Practice Guideline Panel, Staff, and Consortium Representatives. A clinical practice guideline for treating tobacco use and dependence: a US Public Health Service report. JAMA 2000; 283: $3244-3254$

3 Arzneimittelkommission der Deutschen Ärzteschaft. Evidenzbasierte Therapieleitlinien zur Tabakabhängigkeit. Köln: Dt. Ärzteverlag, 2002: 249-276

${ }^{4}$ Holm KJ, Spencer CM. Bupropion - A review of its use in the management of smoking cessation. Drugs 2000; 59(4): 1007-1024

${ }^{5}$ Hurt RD, SACHS DPL et al. A comparison of sustained-release bupropion and placebo for smoking cessation. N Engl J Med 1997; 337: $1195-1202$

${ }^{6}$ Jorenby DEJ, Leischow SJ et al. A controlled trial of sustained-release Bupropion, a nicotine patch, or both for smoking cessation. N Engl J Med 1999; 340: 685-691

${ }^{7}$ Tonnesen $\mathrm{P}$, Norregaard $\mathrm{J}$ et al. Recycling with nicotine patches in smoking cessation. Addiction 1993; 88: 533-539

${ }^{8}$ Gourlay SG, Forbes A et al. Double blind trial of repeated treatment with transdermal nicotine for relapsed smokers. BMJ 1995; 311: $363-366$

${ }^{9}$ Hughes JR, Grass JA, Pillitteri JL. Treatment resistant smokers: a pilot study of nicotine nasal spray and inhaler. J Addict Dis 2000; 19: $95-100$

${ }^{10}$ Hughes JR. Tobacco withdrawal in self-quitters. J Consult Clin Psychol 1992; 60: 689-697

${ }^{11}$ Gonzales DH, Nides MA et al. Bupropion SR as an aid to smoking cessation in smokers treated previously with bupropion: A randomized placebo-controlled study. Clin Pharmacol Ther 2001; 69: 438 - 444

12 Dunner DL, Zisook S et al. A prospective safety surveillance study for Bupropion sustained-release in the treatment of depression. J Clin Psychiatry 1998; 59: $366-373$

13 Tonstad S, Farsang C et al. Bupropion SR for smoking cessation in smokers with cardiovascular disease: a multicentre, randomised study. Eur Heart J 2003; 24: 946-955

14 Tashkin DP, Kanner R et al. Smoking cessation in patients with chronic obstructive pulmonary disease: a double-blind, placebo-controlled, randomised trial. Lancet 2001; 357: 1571-1575

${ }^{15}$ Tsoh JY, Humfleet GL et al. Development of major depression after treatment for smoking cessation. Am J Psychiatry 2000; 157: 368 - 374 